\title{
Bandgap widening by disorder in rainbow metamaterials
}

Cite as: Appl. Phys. Lett. 114, 091903 (2019); https://doi.org/10.1063/1.5081916

Submitted: 17 November 2018 . Accepted: 11 February 2019 . Published Online: 06 March 2019

Paolo Celli (iD), Behrooz Yousefzadeh (iD, Chiara Daraio (iD), and Stefano Gonella
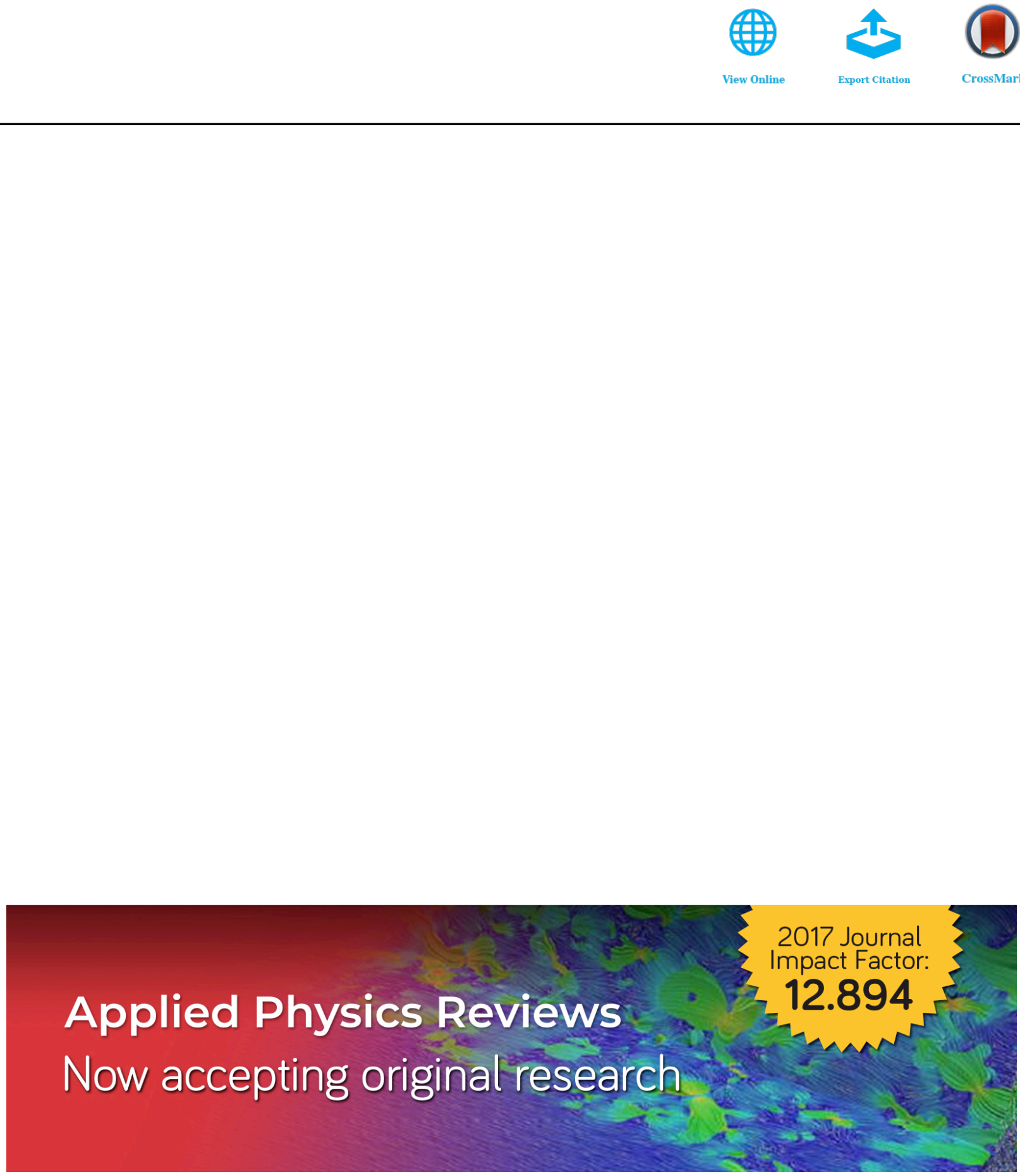


\title{
Bandgap widening by disorder in rainbow metamaterials
}

\author{
Cite as: Appl. Phys. Lett. 114, 091903 (2019); doi: 10.1063/1.5081916 \\ Submitted: 17 November 2018 - Accepted: 11 February 2019 . \\ Published Online: 6 March 2019
}

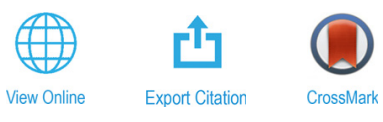

\author{
Paolo Celli, ${ }^{1,2}$ (D) Behrooz Yousefzadeh, ${ }^{2}$ (D) Chiara Daraio, ${ }^{2}$ (D) and Stefano Conella ${ }^{1, a)}$
}

\begin{abstract}
AFFILIATIONS
'Department of Civil, Environmental, and Geo- Engineering, University of Minnesota, Minneapolis, Minnesota 55455, USA

${ }^{2}$ Division of Engineering and Applied Science, California Institute of Technology, Pasadena, California 91125, USA
\end{abstract}

${ }^{a)}$ Electronic mail: sgonella@umn.edu

\begin{abstract}
Stubbed plates, i.e., thin elastic sheets endowed with pillar-like resonators, display subwavelength, locally resonant bandgaps that are primarily controlled by the intrinsic resonance properties of the pillars. In this work, we experimentally study the bandgap response of a tunable heterogeneous plate endowed with reconfigurable families of pillars. We demonstrate that, under certain circumstances, both the spectrum of resonant frequencies of the pillars and their spatial arrangement influence the filtering characteristics of the system. Specifically, both spatially graded and disordered arrangements result in bandgap widening. Moreover, the spectral range over which attenuation is achieved with random arrangements is on average wider than the one observed with graded configurations.
\end{abstract}

Published under license by AIP Publishing. https://doi.org/10.1063/1.5081916

Due to their peculiar mesoscale architectures, metamaterials are capable of manipulating waves in the subwavelength regime ${ }^{1-6}$ - when the wavelengths are much larger than the characteristic microstructural length scales of the medium. These phenomena are typically achieved via homogeneous arrays of resonators, but some effects require the coexistence of heterogeneous populations of resonators with different spectral characteristics. For example, subwavelength waveguiding has been achieved by frequency upshifting selected resonators along a desired waveguide path, ${ }^{4,7-9}$ and topological effects have been observed in systems comprising hexagonal arrangements of different resonator types. ${ }^{10,11}$ Similarly, rainbow trapping requires arrays of resonators with different characteristics, where each type of resonator distills a selected frequency from a broadband input signal. ${ }^{12-19}$ Heterogeneity introduces an additional degree of freedom for metamaterial design that stems from the spatial arrangement of the resonating units. Determining whether this spatial arrangement has any influence on the wave control capabilities of the system requires understanding how neighboring resonators are coupled by the wavecarrying substrate medium. If such an influence is indeed observed, it is of practical interest to determine which spatial configuration maximizes the desired effect.

In this work, we study the effects of the spatial arrangement of local resonators on the bandgap characteristics of an elastic metamaterial, with special emphasis on bandgap widening. Our medium of choice is a stubbed plate in which the plate substrate behaves as the wave-carrying medium, and arrays of surface pillars act as resonators $^{20-28}$ (Fig. 1). It is documented that graded arrangements of heterogeneously tuned resonators display wider bandgaps than their homogeneous counterparts. ${ }^{14}$ Here, our aim is to highlight the role played by the compliance of the wave-carrying medium in controlling the bandgap response. When the plate is significantly more compliant than the pillars, the wave transmission characteristics become more sensitive to the spatial arrangement of the resonators. Thus, even heterogeneous arrangements that differ from each other solely in terms of their spatial characteristics result in appreciably different bandgap responses. Specifically, we intend to show that random arrangements systematically widen the bandgaps with respect to nominally similar graded configurations.

In order to achieve the versatility required to test multiple architectures within a single reconfigurable specimen, we design a modu$\operatorname{lar}^{29-31}$ stubbed plate with tunable resonators. We arrange arrays of LEGO $^{\circledR}$ bricks on a thin baseplate strip (Fig. 1), following a testing paradigm that we previously introduced for homogeneous locally resonant phononic crystals. ${ }^{28}$ The specimen features $12 \times 5$ pillars arranged according to a square lattice in the central section of the strip. Each resonator can assume one of the six discrete inertial configurations shown at the bottom-right of Fig. 1, obtained by sliding the conical tip down the rod by discrete increments of $\delta h=3.25 \mathrm{~mm}$. This manual tuning alters the effective inertial characteristics of a pillar and thereby its natural frequencies, and is conceptually similar to strategies 


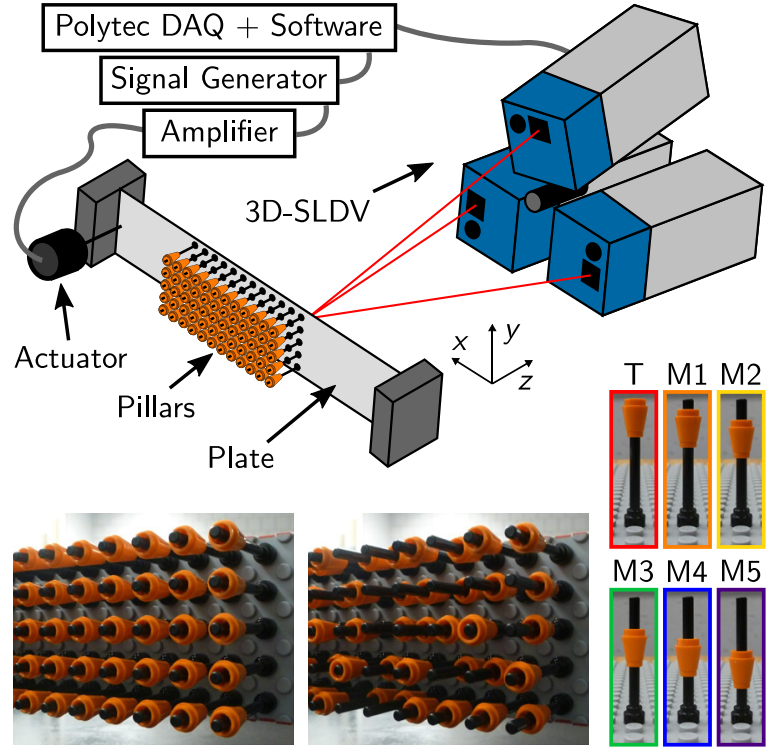

FIG. 1. Experimental setup (top). The bottom images illustrate two configurations achievable through different selections and arrangements of the six pillar types shown on the right (T-M5), obtained by sliding the conical brick down the rod by discrete increments.

discussed by other authors for Bragg bandgap tunability. ${ }^{32-34}$ Two examples of different pillar arrangements are shown at the bottom of Fig. 1. The plates are excited with a pseudorandom waveform prescribed by a shaker to establish standing flexural wave patterns over a

broad spectrum of frequencies, and their out-of-plane response is recorded with a 3D Scanning Laser Doppler Vibrometer (3D-SLDV). More details on the setup are discussed in the supplementary material section.

The effect of introducing arrays of pillars is to open locally resonant bandgaps in the phonon band structure. This is clear when comparing the dispersion relation (reconstructed from experimental data) of the bare baseplate, which features a single flexural mode in the range of interest, against that of the baseplate with all pillars of the M1 type, where the mode is split and a hybridization gap arises between 242 and $311 \mathrm{~Hz}$ [Figs. 2(a) and 2(b)]. Here, the dotted lines that follow the dispersion branches are obtained by tracking the maxima of the spectral amplitude (the underlying grayscale colormap). More details on the band diagram reconstruction are given in the supplementary material. The flexural deflection shapes recorded along the strip's centerline [Fig. 2(c)] reveal some peculiar aspects of the physical mechanisms responsible for this gap. These observations are crucial to properly interpret the results discussed in the rest of this work. It can be noticed that, at the onset of the gap, the region of the plate below the pillars undergoes flexural deformation, whose amplitude is not negligible with respect to the maximum plate deflection and with respect to the motion of the pillars. This suggests that, in our system, what effectively resonates are not the pillars taken as stand-alone beam-like structural elements forced at their base (as in other works featuring similar platforms ${ }^{26,35}$ ) but rather the whole unit cell comprising the plate and the pillar. In particular, each pillar's main contribution is to add rotatory inertia to one resonating unit. We conjecture that this resonant behavior is due to the specific landscape of mechanical properties in our specimen, where the plate has low relative stiffness with respect to the pillars, which establishes strong coupling

(c)

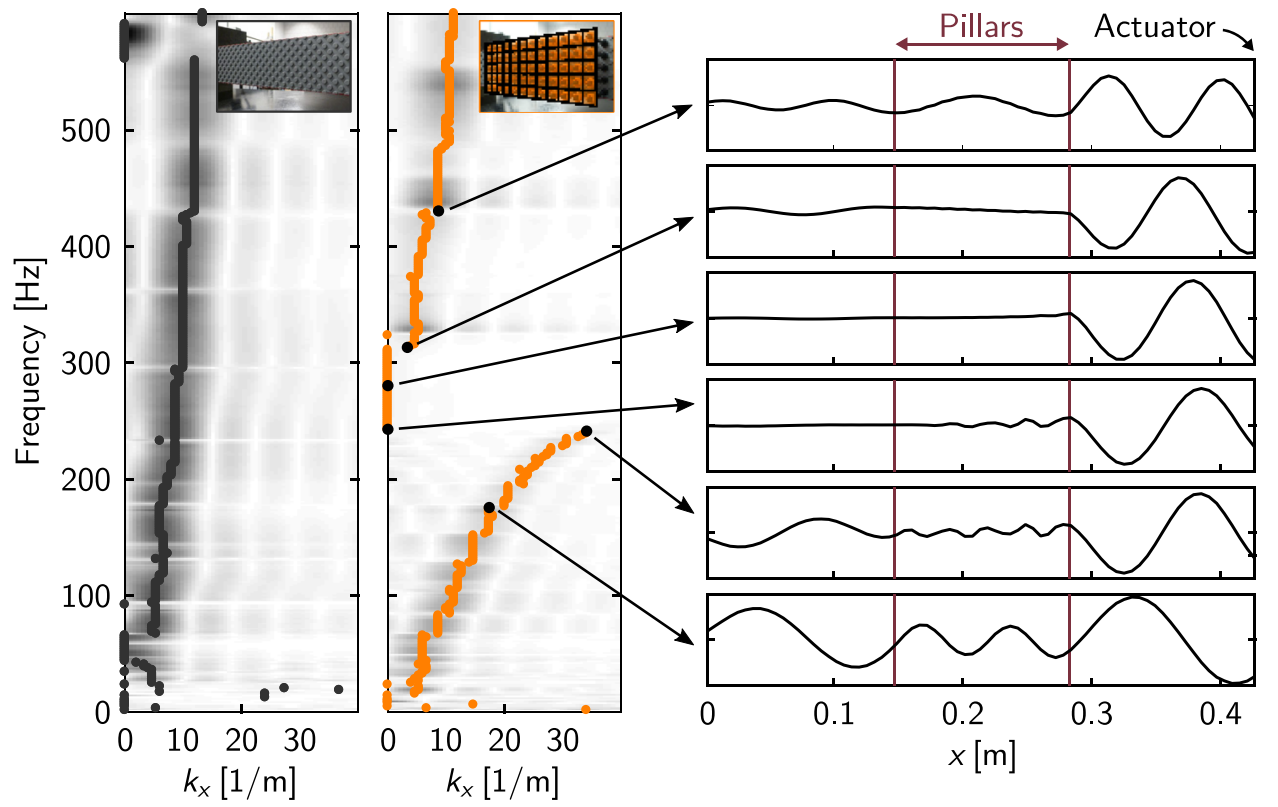

(d)

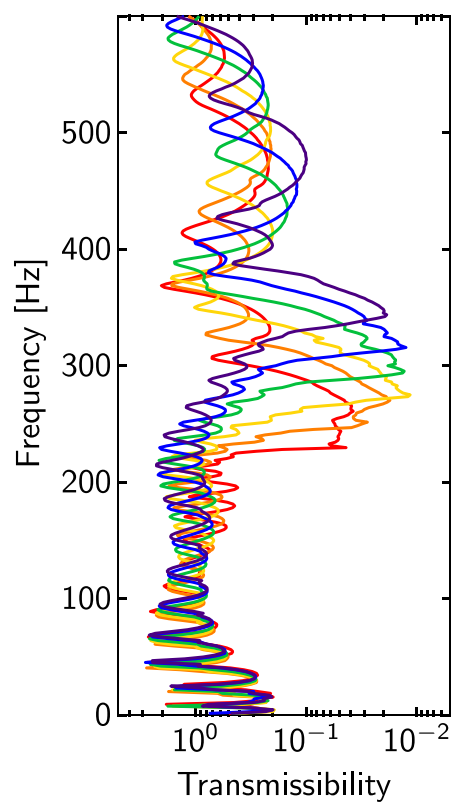

FIG. 2. (a) and (b) Experimentally reconstructed dispersion relations ( $k_{x}$ is the wave number along the $x$-direction) of the bare baseplate and of the baseplate with $5 \times 12$ identical M1 pillars, respectively. (c) Deformed shapes for the (b) case, measured at the centerline of the strip for frequencies below, inside, and above the gap. (d) Transmissibilities of specimens of identical pillars, matching the six types color-coded in Fig. 1. 
between neighboring pillars through the plate substrate. For completeness, we analyzed the response of different uniform configurations, each featuring one of all the possible resonator types. The measured transmissibilities indicate a trend of shifted and partially overlapping bandgaps [Fig. 2(d)]. As the tip mass is slid down the pillars and their effective inertia is decreased, the onsets of the bandgaps shift towards higher frequencies (see dispersion curves in the supplementary material section).

Locally resonant bandgaps are usually narrow and therefore impractical to design mechanical filters that are effective against broadband excitations. This limitation has inspired numerous widening strategies, among which we recall trampoline effects, ${ }^{36}$ rainbow trapping, ${ }^{14,37}$ bandgap adjoining, ${ }^{38,39}$ and disorder-based methods involving Anderson localization phenomena. ${ }^{40-42}$ Here, we investigate the behavior of heterogeneous populations of resonators with emphasis on the dependence of the bandgap width upon the spatial arrangement of the resonators. To this end, we compare the performance of three classes of configurations: (i) uniform arrangements, (ii) graded arrangements of heterogeneous resonators, and (iii) random populations of heterogeneous resonators. The measured transmissibilities of a few representative configurations are shown in Fig. 3. In each subfigure, the thick black line represents the transmissibility for the arrangement shown in the corresponding inset; all the configurations feature 10 resonators of each type-T, M1, M2, M3, M4, and M5 denoted by the color coding introduced in Fig. 1. The results for two graded architectures with different gradient patterns [the pattern in Fig. 3(a), called graded A, and the one in Fig. 3(b), labeled graded B] clearly highlight a widening of the bandgap with respect to their monochromatic counterparts. In both cases, the total bandgap spans the frequency interval encompassing the individual gaps of three monochromatic configurations. This result is a manifestation of the rainbow trapping effect. ${ }^{14-16}$ Interestingly, we observe that the graded A configuration produces a wider and deeper gap than the graded B one, suggesting that the performance of graded architectures is influenced by the period of the spatial arrangement. The transmissibility of a representative spatially disordered arrangement (MATLAB-generated) is shown in Fig. 3(c). The most distinctive morphological difference brought about by randomization is that the bandgap is wider than its graded and homogeneous counterparts-stretching here over the frequency interval spanned by five individual bandgaps. The reliability of this observation is confirmed by averaging three random realizations [Fig. 3(d), black line]. These experimental results (later corroborated by numerical results) lead to the conclusion that randomization causes attenuation over a wider frequency range, but this comes at the expense of the attenuation amplitude, which decreases with respect to the graded case. A similar effect is well documented in non-resonant disordered systems. ${ }^{43}$

To substantiate our experimental findings and to explore the robustness of these results against variations in the characteristics of plate and pillars, we perform finite element simulations, carried out in Abaqus/Standard. The plate is discretized using 3D shell elements. The pillars are modeled as Timoshenko beams that are assumed to be perfectly anchored to the plate at a single point. The conical sliding tip is assumed to be a point mass. The numerical model captures all the important qualitative features of the experimental results, albeit without matching quantitatively the frequencies of the experimental bandgaps. For example, the dispersion relation and the transmissibility curve [dashed gray lines and orange line in Fig. 4(a), respectively] for
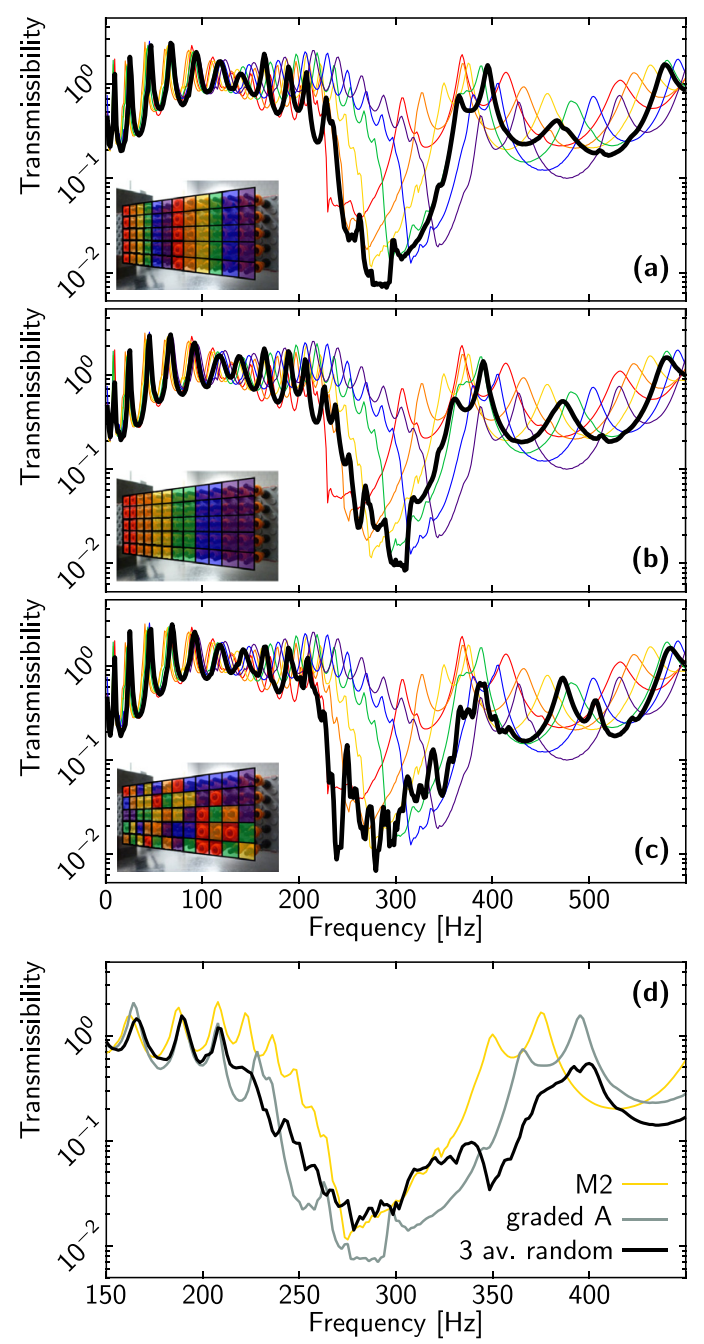

FIG. 3. Influence of the spatial arrangement of resonators on wave attenuation. The resonators are divided into six groups of ten units each, implementing the six configurations shown in Figs. 1(a) and 1(b). Transmissibilities for graded configurations [the pattern in (a) is called graded A and the one in (b) graded B] and (c) spatially randomized configurations, marked by thick black lines; thin color-coded lines refer to monochromatic configurations. (d) Comparison of bandgaps for uniform, graded and random (3 averages) configurations.

the M1 homogeneous configuration feature trends that closely resemble the experimental results in Figs. 2(b) and 2(d). The deflection shape at the onset of the bandgap [inset in Fig. 4(a)] highlights that the resonance is characterized by an undulatory motion of the plate, also consistent with the experiments [Fig. 2(c)]. An advantage of numerical simulations is that we can compare a significantly larger set of realizations of the disordered metamaterial to infer the statistical behavior of the ensemble. Figure 4(b) shows the average transmissibility curve obtained by averaging 150 disordered realizations. These results corroborate the previous conclusion [Fig. 3(d)] that the locally resonant bandgap of the random configuration is on average wider than the bandgap of the graded configurations. More detailed comparisons are provided in the supplementary material. 

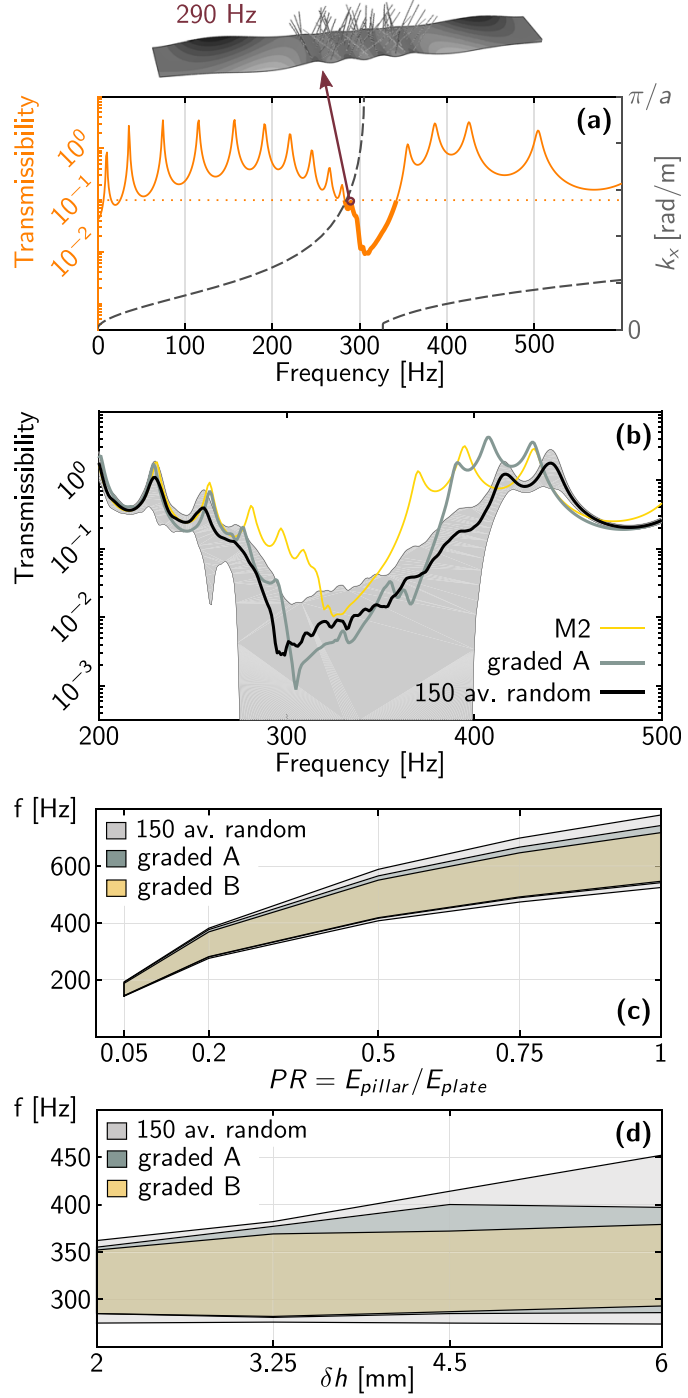

FIG. 4. Numerical simulations. (a) Dispersion relation (dashed gray line) and transmissibility (orange line) for the M1 homogeneous configuration. We consider everything below the dotted orange line (transmissibility $<10^{-1}$ ) as bandgap. The inset depicts a deflection shape right before the bandgap onset. (b) Comparison of bandgaps for uniform, graded and random (average of 150 realizations) configurations. The gray area is the standard deviation of the black curve. (c) and (d) Bandgap width as a function of the plate ratio $(P R)$, and of the increment by which the mass is slid down the pillars $(\delta h)$, respectively. Default values are $\delta h$ $=3.25 \mathrm{~mm}, P R=0.2$.

We leverage the numerical platform to further explore the influence of some key system parameters on the bandgap behavior. In particular, we explore the effect of (i) the relative stiffness between the plate and pillars and (ii) the spacing between the resonance frequencies of the pillars. For this parametric study, we focus on the bandgap width, specifically on how it compares between the random and the two graded configurations. Figure 4(c) shows that stiffening the plate (as parameterized by $P R$, the ratio between the elastic modulus of the pillar and that of the plate) will widen the bandgap for all three configurations, as well as shift them towards higher frequencies. The latter aspect provides further evidence that the plate is part of the resonating unit. Figure 4(d) shows the bandgap width as a function of $\delta h$, which is the increment by which the conical mass is slid down the pillars ( $\delta h$ was set to the default 3.25 value in the previous discussion). Increasing $\delta h$ results in a wider separation between the bandgaps of the six configurations made of uniform pillar arrangements and therefore widens the total bandgaps for both the graded and random configurations. ${ }^{44}$ However, it is clear that the widening effect is more pronounced for random configurations. It is nonetheless noted that, although random configurations have the widest bandgap on average, the widening does not necessarily occur for all realizations, as evidenced by the standard deviation [gray shading in Fig. 4(b)]. From this parametric analysis, we conclude that the disorderinduced widening of the bandgap is robust to small changes in structural and material properties of the metamaterial and occurs over a wider frequency range than the bandgap produced by grading the resonators.

In conclusion, this work takes advantage of highly reconfigurable LEGO $^{\circledR}$-brick-based stubbed plates to highlight the potential advantages of mechanical metamaterials featuring heterogeneous populations of resonators as broadband filters and showcases the importance of the spatial arrangement of the resonators. In particular, we have realized and tested a tunable elastodynamic rainbow trap and we have shown that the interplay between heterogeneity in the resonators' characteristics and spatial disorder results in a widening of the filtering effects compared to the more conventional spatially ordered rainbow material concepts.

See supplementary material for a more detailed account on the experimental setup, the numerical model, and for additional results.

S.G. and P.C. acknowledge support from the National Science Foundation (CMMI-1266089). P.C. acknowledges support from the University of Minnesota Doctoral Dissertation Fellowship. B.Y. acknowledges support from the Natural Science and Engineering Research Council of Canada through a postdoctoral fellowship. C.D. and B.Y. acknowledge partial support from the National Science Foundation (EFRI-1741565). We thank W. Zhang, N. Bausman, M. Turos, A. Palermo, V. Tournat, and A. Cebrecos for their help and fruitful discussions.

\section{REFERENCES}

'Z. Liu, X. Zhang, Y. Mao, Y. Y. Zhu, Z. Yang, C. T. Chan, and P. Sheng, Science 289, 1734 (2000).

${ }^{2}$ J. Zhu, J. Christensen, J. Jung, L. Martin-Moreno, X. Yin, L. Fok, X. Zhang, and F. J. Garcia-Vidal, Nat. Phys. 7, 52 (2011).

${ }^{3}$ S. Zhang, C. Xia, and N. Fang, Phys. Rev. Lett. 106, 024301 (2011).

${ }^{4}$ F. Lemoult, N. Kaina, M. Fink, and G. Lerosey, Nat. Phys. 9, 55 (2013).

${ }^{5}$ A.-C. Hladky-Hennion, J. O. Vasseur, G. Haw, C. Cronne, L. Haumesser, and A. N. Norris, Appl. Phys. Lett. 102, 144103 (2013).

${ }^{6}$ H. Zhu and F. Semperlotti, Phys. Rev. Lett. 117, 034302 (2016).

${ }^{7}$ M. Addouche, M. A. Al-Lethawe, A. Elayouch, and A. Khelif, AIP Adv. 4, 124303 (2014).

${ }^{8}$ Z. Gao, F. Gao, and B. Zhang, Appl. Phys. Lett. 108, 041105 (2016).

${ }^{9}$ N. Kaina, A. Causier, Y. Bourlier, M. Fink, T. Berthelot, and G. Lerosey, Sci. Rep. 7, 15105 (2017).

${ }^{10}$ R. K. Pal and M. Ruzzene, New J. Phys. 19, 025001 (2017).

${ }^{11}$ R. Chaunsali, C.-W. Chen, and J. Yang, New J. Phys. 20, 113036 (2018). 
${ }^{12}$ K. L. Tsakmakidis, A. D. Boardman, and O. Hess, Nature 450, 397 (2007).

${ }^{13}$ J. Zhu, Y. Chen, X. Zhu, F. J. Garcia-Vidal, X. Yin, W. Zhang, and X. Zhang, Sci. Rep. 3, 1728 (2013).

${ }^{14}$ S. Krödel, N. Thomé, and C. Daraio, Extreme Mech. Lett. 4, 111 (2015).

${ }^{15}$ D.-G. Zhao, Y. Li, and X.-F. Zhu, Sci. Rep. 5, 9376 (2015).

${ }^{16}$ D. Cardella, P. Celli, and S. Gonella, Smart Mater. Struct. 25, 085017 (2016).

${ }^{17}$ Z. Tian and L. Yu, Sci. Rep. 7, 40004 (2017).

${ }^{18}$ A. Colombi, V. Ageeva, R. J. Smith, A. Clare, R. Patel, M. Clark, D. Colquitt, P. Roux, S. Guenneau, and R. V. Craster, Sci. Rep. 7, 6750 (2017).

${ }^{19}$ Y. Guo, M. Hettich, and T. Dekorsy, New J. Phys. 19, 013029 (2017).

${ }^{20}$ T.-T. Wu, Z.-G. Huang, T.-C. Tsai, and T.-C. Wu, Appl. Phys. Lett. 93, 111902 (2008).

${ }^{27}$ Y. Pennec, B. Djafari-Rouhani, H. Larabi, J. O. Vasseur, and A. C. HladkyHennion, Phys. Rev. B 78, 104105 (2008).

${ }^{22}$ M. Oudich, M. Senesi, M. B. Assouar, M. Ruzenne, J.-H. Sun, B. Vincent, Z. Hou, and T.-T. Wu, Phys. Rev. B 84, 165136 (2011).

${ }^{23}$ M. B. Assouar, M. Senesi, M. Oudich, M. Ruzzene, and Z. Hou, Appl. Phys. Lett. 101, 173505 (2012)

${ }^{24}$ F. Casadei, T. Delpero, A. Bergamini, P. Ermanni, and M. Ruzzene, J. Appl. Phys. 112, 064902 (2012).

${ }^{25}$ Y. Achaoui, V. Laude, S. Benchabane, and A. Khelif, J. Appl. Phys. 114, 104503 (2013).

${ }^{26}$ M. Rupin, F. Lemoult, G. Lerosey, and P. Roux, Phys. Rev. Lett. 112, 234301 (2014).

${ }^{27}$ R. Pourabolghasem, A. Khelif, S. Mohammadi, A. A. Eftekhar, and A. Adibi, J. Appl. Phys. 116, 013514 (2014).
${ }^{28}$ P. Celli and S. Gonella, Appl. Phys. Lett. 107, 081901 (2015).

${ }^{29}$ Z. Wu, R. L. Harne, and K. W. Wang, J. Intell. Mater. Syst. Struct. 27, 1189 (2016).

${ }^{30}$ S. Lee, B. Kang, H. Keum, N. Ahmed, J. A. Rogers, P. M. Ferreira, S. Kim, and B. Min, Sci. Rep. 6, 27621 (2016).

${ }^{31}$ G. Memoli, M. Caleap, M. Asakawa, D. R. Sahoo, B. W. Drinkwater, and S. Subramanian, Nat. Commun. 8, 14608 (2017).

${ }^{32}$ C. Goffaux and J. P. Vigneron, Phys. Rev. B 64, 075118 (2001).

${ }^{33}$ V. Romero-Garcia, C. Lagarrigue, J.-P. Groby, O. Richoux, and V. Tournat, J. Phys. D: Appl. Phys. 46, 305108 (2013).

${ }^{34} \mathrm{M}$. Thota, S. Li, and K. W. Wang, Phys. Rev. B 95, 064307 (2017).

${ }^{35}$ E. G. Williams, P. Roux, M. Rupin, and W. A. Kuperman, Phys. Rev. B 91, 104307 (2015).

${ }^{36}$ O. R. Bilal and M. I. Hussein, Appl. Phys. Lett. 103, 111901 (2013).

${ }^{37}$ A. Banerjee, R. Das, and E. P. Calius, J. Appl. Phys. 122, 075101 (2017).

${ }^{38}$ E. Coffy, T. Lavergne, M. Addouche, S. Euphrasie, P. Vairac, and A. Khelif, J. Appl. Phys. 118, 214902 (2015).

39. H. Oh, H. M. Seung, and Y. Y. Kim, Appl. Phys. Lett. 108, 093501 (2016).

${ }^{40}$ O. Thorp, M. Ruzzene, and A. Baz, Smart Mater. Struct. 10, 979 (2001).

${ }^{41}$ R. Sainidou, N. Stefanou, and A. Modinos, Phys. Rev. Lett. 94, 205503 (2005).

${ }^{42}$ O. Richoux, E. Morand, and L. Simon, Ann. Phys. 324, 1983 (2009).

${ }^{43}$ Y.-K. Lin, Appl. Mech. Rev. 49, 57 (1996).

${ }^{44} \mathrm{An}$ alternative to increasing $\delta h$ is increasing the value of the conical mass that slides along each beam. We found this to have a qualitatively similar effect to increasing $\delta h$. 